\title{
A TEST PARTICLE APPROACH IN THE STOCHASTIC STABILITY ANALYSIS OF CHARGED PARTICLE BEAMS
}

\author{
Javier VITELA E. \\ Instituso de Ciencias Nucleares. Universidad Nacional Autonoma de Mcxico, 04.510 Mixico. \\ D.F. Mevico
}

\author{
A. Ziya AKCASU \\ Depurmem of Nuclear Engineering. The University of Michuan. Ann Arbor. MI HSIUM, USA
}

Received 18 April 1989

Revised manuscript received 31 July 1989

\begin{abstract}
The stochastic stability of a charged particle beam propagating in a randomly unhomogeneous medum is analyzed using an internal test particle approach. The effect of the random medium is included by adding a stochastic fluctuating term in the neutralization factors. The effects of weakly colored noise and of fluctuation, called telegraphic nome, 1 i imo valued Markov process, are studied. The exact solution of the telegraphut none an compared with the approximate solution corresponding to the weakly colored nowe. which was obtaned using the Stratonovich approdch. by means of an expanson technique it is concluded that the heam is dadys probabilistically unstable. wheneser the power spectral denoty of the fluctuations contans a component at the frequency of the radial ancillations of the tert particles.
\end{abstract}

\section{Introduction}

The motion of a test particle inside an infinitely long. cylindrically symmetric relativistic non-neutral charged particle beam propagating in a plasma medium parallel to an uniform external magnetic field is governed by: the radial electric field produced by the beam space charge; the self-pinching force due to the beam current; the external magnetic field: as well as the degree in which the medium is able to neutralize the beam current and charge [1,2]. The effect of the medium on the motion of the test particle is usually taken into account by parameters in the equations of motion called space charge and current neutralization factors. In the standard analysis these parameters are assumed constant: however, when the medium is randomly unhomogeneous, due to fluctuations 
produced by turbulence, the neutralization factors are not constant anymore and in general they become random functions of time.

The fluctuations in a physical system may have different origins. One type of fluctuations occurring in a beam-plasma systen: are those produced by the microscopic nature of the collision processes that take place. In this case, if it can be assumed that the velocity of the test particle in the beam constitutes a Markov process. $\{\boldsymbol{v}(t)\}$, then the conditional probability density function $P\left(v, t \mid v_{0}, 0\right)$ satisfies a Chapman-Kolmogorov equation and is therefore completely specified by its transition probability per unit time. This type of fluctuations are usually referred to as internal noise, since these are an intrinsic part of the evolution mechanism of the system, and cannot be eliminated. If we could solve the master equation exactly the Markov process $\{\boldsymbol{v}(t)\}$ would be completely known; this is not possible in general, and one has to use approximate techniques to obtain the solution. In some special cases [3-6]. techniques have been developed to study the evolution of systems with internal noise.

On the other hand, when a charged particle beam is moving in a plasma, many types of linear and nonlinear instabilities may develop which lead to turbulence in the system. The rigorous treatment of this problem consists in studying the behavior of the beam-plasma system self-consistently; this means that the power spectral density of the fluctuations produced by the turbulence that affect the behavior of the system, should be calculated using the spectrum of the instabilities developed by the system itself. This is in general a formidable task and some approximations should be done in order to gain some insight to the problem.

In many cases a simplified approach is used, which consists in assuming that the spectrum of fluctuations is known, and then the behavior of the system in such a turbulent medium is analyzed. In this approximation the perturbations are thought to be an external source of noise coupled to a noiseless system. This type of noise is assumed to be independent of the system and not affected by it [5].

This latter approach is the one used in this work. To further simplify the problem we restrict ourselves to the study of the motion of test particles inside the beam, when random perturbations in the medium in which it propagates are present. Thus, the equation of motion of the test particies inside the beam will be governed by stochastic differential equations, i.e. differential equations whose parameters contain random functions of time [7]. Some of the stochastic fluctuations generally assumed in the study of physical systems subject to external noise and which are used in this work are: Gaussian white noise; arbitrary fluctuations with short correlation times, i.e. weakly colored noise; and a two valued Markov process, also called telegraphic noise. 
Dynamical systems driven by white noise have been subject to a controversy, which has been referred to as the Itô-Stratonovich dilemma $[8,9]$. However, Gray ex al. [10], Mortensen [11], and van Kampen [12] have concluded that, although both approaches are mathematically correct, Stratonovich rules are better suited for the study of physical systems subject to white noise, since this type of noise is only a mathematical idealization. Hence, in this work, the white noise results are obtained from the Stratonovich results for weakly colored noise as a limiting case.

This paper has been organized as follows: Section 2 describes the physical model adopted here for the description of the motion of the test particles in the beam, together with some of the characteristics of their behavior when the neutralization factors contain random fluctuations. In section 3 , the case of weakly colored noise is studied using the Stratonovich approach [9, 13]: the stability in the mean and in the mean squared is analyzed using the RouthHurwitz criterion [14]. As a particular case, the white noise and its implications is also discussed in this part. Finally, in section 4, the case of binary telegraphist signal perturbations is considered and the exact solution is obtained. Comparison of this exact solution, valid regardless of the magnitude and correlation time of the fluctuations, with the corresponding approximate solution obtained with the Stratonovich approach is done following an expansion technique.

\section{The physical model}

In order to simplify the analysis, we assume that the beam particles do not suffer from collisions and move along the $z$-direction with relativistic constant velocity $v_{z}$; the transverse motion of the particles is taken to be not relativistic. By doing this, we neglect the effect of the radial motion of the particles in the $z$-component of the relativistic momentum $m \gamma v$. With these assumptions, the transverse equations of motion of a particle of charge $q$ and rest mass $m$, inside a particle beam moving along a constant uniform external magnetic field $\boldsymbol{B}_{0}=B_{0} \hat{e}_{z}$, are $[1,2]$

$$
\begin{aligned}
& \frac{\mathrm{d}^{2}}{\mathrm{~d} t^{2}} x=-\omega_{1}^{2} x+\frac{\Omega_{0}}{\gamma} \frac{\mathrm{d} y}{\mathrm{~d} t}, \\
& \frac{\mathrm{d}^{2}}{\mathrm{~d} t^{2}} y=-\omega_{1}^{2} y-\frac{\Omega_{0}}{\gamma} \frac{\mathrm{d} x}{\mathrm{~d} t},
\end{aligned}
$$

where

$$
\omega_{1}^{2} \equiv \omega_{\mathrm{p}}^{2}\left[f_{\mathrm{q}}-1+\beta^{2}\left(1-f_{\mathrm{m}}\right)\right] / 2 \gamma,
$$


$\Omega_{0} \equiv q B_{0} / m c, \omega_{\mathrm{p}}^{2} \equiv 4 \pi q^{2} n / m, \gamma \equiv\left(1-\beta^{2}\right)^{-1 / 2}$, and $\beta \simeq v_{z} / c$; here, $c$ is the speed of light, $n$ is the particle density of the beam. and $f_{4}$ and $f_{\mathrm{m}}$ are the space charge and current neutralization factors, respectively. In what follows we will assume $\gamma$ is constant.

It is easily seen from eqs. (1) that the condition for bounded solutions or neutral stability is

$$
\omega_{0}^{2}>0
$$

where

$$
\omega_{0}^{2} \equiv\left(\frac{\Omega_{0}}{2 \gamma}\right)^{2}+\omega_{1}^{2}
$$

By defining the complex variable $S=x+i y$, eqs. (1) can be written as a single equation, i.e.

$$
\frac{\mathrm{d}^{2} S}{\mathrm{~d} t^{2}}+\mathrm{i} \frac{\Omega_{0}}{\gamma} \frac{\mathrm{d} S}{\mathrm{~d} t}+\omega_{1}^{2} S=0
$$

In $r-\theta$ coordinates, the equation of motion is expressed as

$$
\frac{\mathrm{d}^{2} R}{\mathrm{~d} t^{2}}-\left\{\omega^{2}(R)+\frac{\Omega_{0}}{\gamma} \omega(R)-\frac{\omega_{\mathrm{p}}^{2}}{2 \gamma}\left[f_{\mathrm{q}}-1+\beta^{2}\left(1-f_{\mathrm{m}}\right)\right]\right\} R=0 .
$$

where $\omega(R) \equiv \mathrm{d} \theta / \mathrm{d} t$, and is obtained from the conservation of the canonical angular momentum per unit mass,

$$
L=\gamma \omega R^{2}+\frac{1}{2} \Omega_{0} R^{2} .
$$

The special case of constant radius trajectories, $R_{\mathrm{c}}$, are obtained requiring that the second term in eq. (5) is equal to zero; this leads to the following allowable angular velocities [2]:

$$
\omega_{ \pm}=-\Omega_{0} / 2 \gamma \pm \omega_{i 1} \text {. }
$$

when $\omega_{11}^{2}>0$. It is easily seen from eqs. (6) and (7) that the angular momentum per unit mass, $L$, is given by

$$
L=\gamma \omega_{0} R_{\mathrm{e}}^{2}
$$

from where it follows that $L$ depends only on the equilibrium radius $R_{\mathrm{e}}$, when 
$\omega_{11}$ is constant, or that the equilibrium radius is determined once $\omega_{11}$ and $L$ are specified.

Solving eq. (6) for $\omega$, and substituting in eq. (5) we obtain the following equation for $R(t)$ :

$$
\frac{\mathrm{d}^{2}}{\mathrm{~d} t^{2}} R+\omega_{10}^{2} R-\frac{L^{2}}{\gamma^{2} R^{3}}=0
$$

the solution of this equation is bounded if $\omega_{n}^{2}>0$. In the phase space $R-\dot{R}$. the trajectories form closed curves that satisfy the following equation:

$$
[\dot{R}]^{2}+\left[\frac{L}{\gamma R}-\omega_{11} R\right]^{2}=K^{2}
$$

where $K$ is the maximum radial velocity of the particle. See fig. 1 Notice that the equilibrium state is obtained only when $K=0$, i.e. $\dot{R}_{\mathrm{e}}=0$ and $R_{\mathrm{c}}=$ $\left(L / \gamma \omega_{10}\right)^{1 / 2}$.

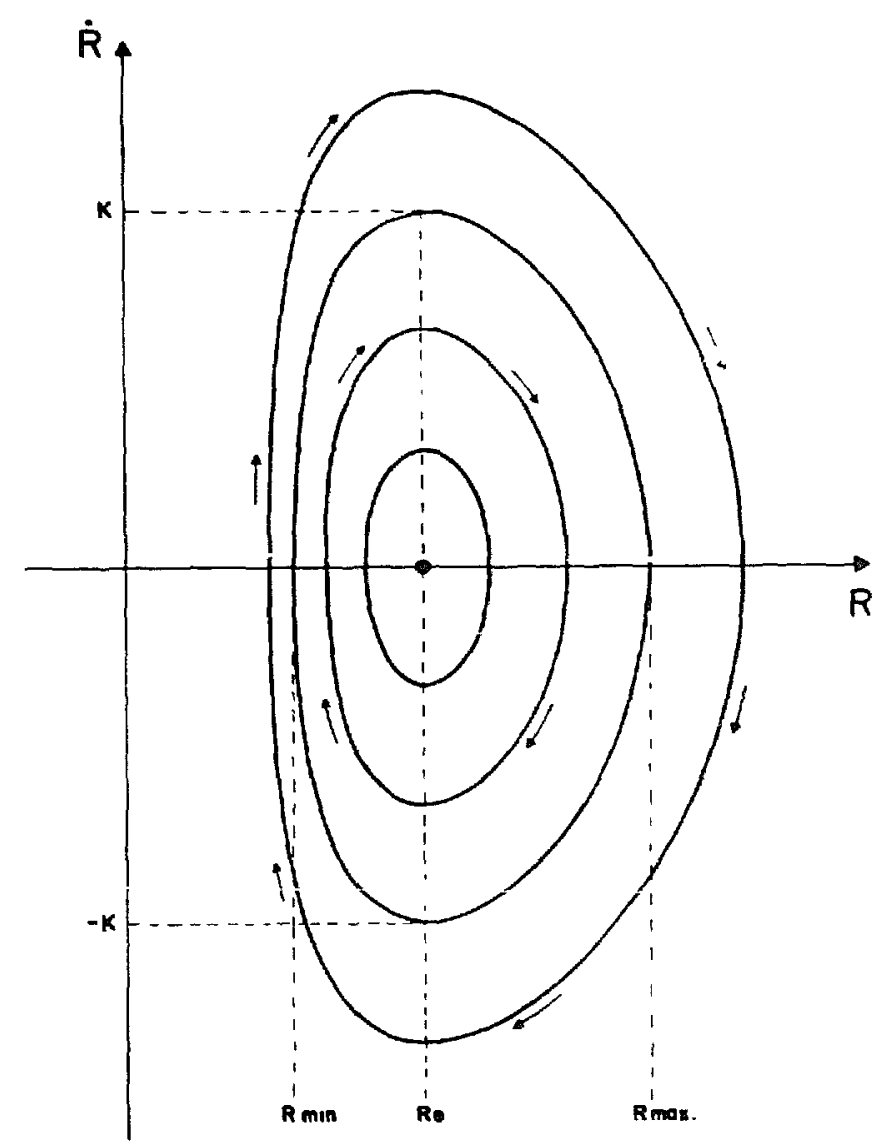

Fig. 1. Typical phase space trajectories in the $R-R$ plane of a test particle in an unperturbed beam. 
If the beam is propagating in a random medium, the neutralization factors are stochastic functions of time; it can be demonstrated that the canonical angular momentum per unit mass, $L$, defined in eq. (6) remains a constant of motion even in the presence of fluctuations in the neutralization factors as long as the external magnetic field $B_{0}$ is constant. Thus, when the neutralization factors $f_{\mathrm{q}}$ and $f_{\mathrm{m}}$ randomly fluctuate, according to eq. (8) the equilibrium radius $R_{\mathrm{c}}$ is also fluctuating, and the test particle trajectories in the phase plane will be a sequence of paths centered around the instantaneous position of the equilibrium state. In fig. 2 we illustrate this for the case of telegraphic noise; as shown in this figure, the subpaths in the phase plane are centered in two different equilibrium points, since $\omega_{0}$ can acquire only two values.

Using the fact that $L$ remains a constant of motion when the neutralization factors are functions of time, yielding $\omega_{0}^{2}$ time dependent, the following exact differential equation for $R^{2}(t)$ can be derived from eq. (9):

$$
\frac{\mathrm{d}^{3}}{\mathrm{~d} t^{3}} R^{2}+4 \omega_{0}^{2} \frac{\mathrm{d}}{\mathrm{d} t} R^{2}+2\left(\frac{\mathrm{d}}{\mathrm{d} t} \omega_{0}^{2}\right) R^{2}=0 ;
$$

if follows from this equation that if the neutralization factors are constant, the square value of the radial position is given by

$$
R^{2}(t)=A_{0}+A_{1} \sin \left(2 \omega_{0} t+\phi\right)
$$

where $A_{0}$ and $A_{1}$ are constants of integration.

We shall point out here that the frequency of the radial oscillations, $2 \omega_{0}$, is independent of both the angular momentum and the maximum radial velocity; in other words, it is determined only by the physical characteristics of the medium in which the beam is moving.

In order to study the influence of random fluctuations in the neutralization factors on the motion of test particles, in the next sections we assume that different types of noise affect the neutralization factors. To determine the stochastic stability we need to analyze the behavior of ine mean position of the test particles, $\langle x\rangle$ and $\langle y\rangle$, as well as the dispersion around this mean position, which is given by $\sigma^{2}=\left\langle(x-\langle x\rangle)^{2}\right\rangle+\left\langle(y-\langle y\rangle)^{2}\right\rangle=\left\langle x^{2}\right\rangle+$ $\left\langle y^{2}\right\rangle-\langle x\rangle^{2}-\langle y\rangle^{2}$. Since the mean square radius is $\left\langle R^{2}\right\rangle=\left\langle x^{2}\right\rangle+\left\langle y^{2}\right\rangle$, the stochastic stability will be determined only by the behavior of the mean position and the mean square radius of the test particles. It is in this sense that we say that, if $\left\langle R^{2}\right\rangle$ grows in time, even if the mean position is bounded, the beam is probabilistically unstable. The physical meaning consists in that the probability of finding a test particle farther and farther away from the center of the beam is always increasing in time even if its mean position is bounded. 

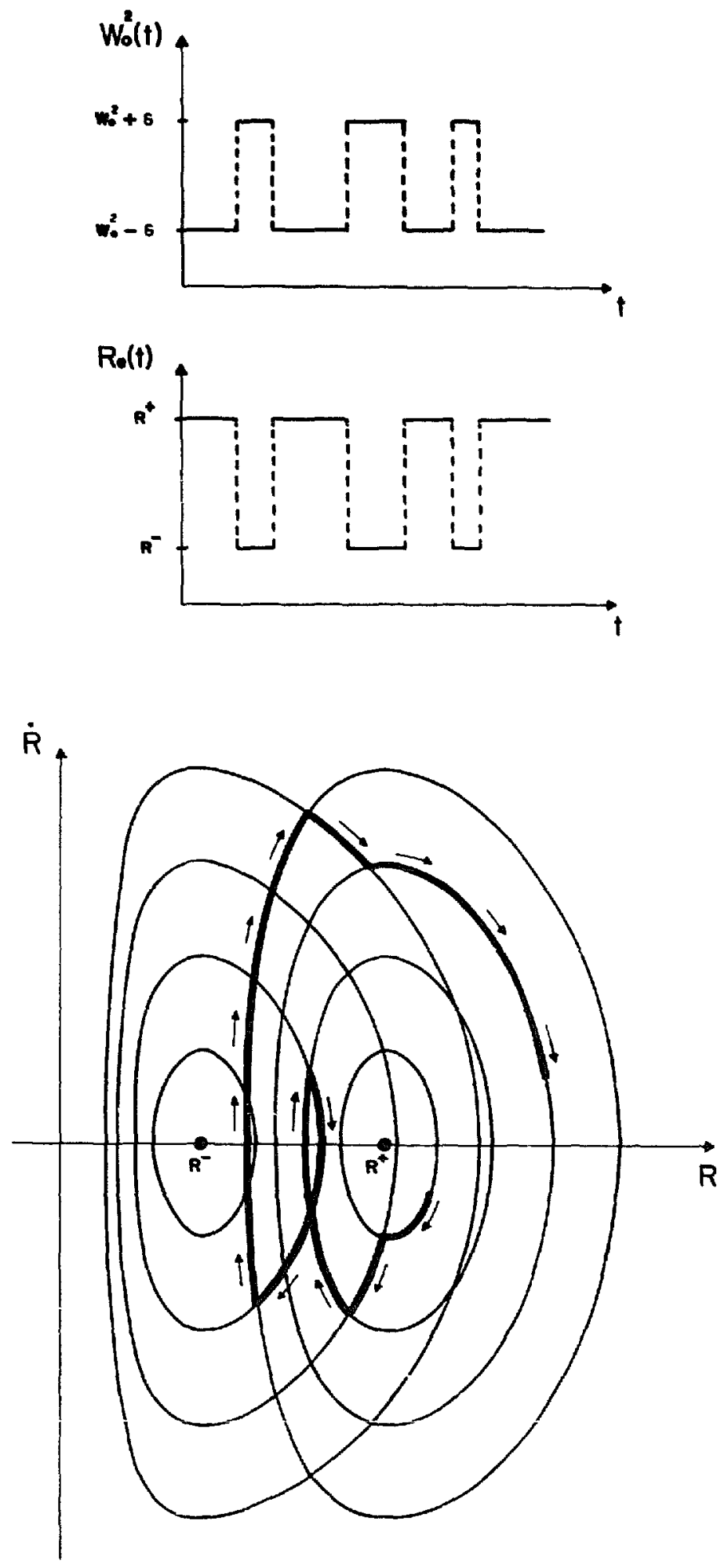

Fig. 2. Phase space trajectories of a test particle when the neutralization factors contan telegraphic noisc Top: Random fluctuations of the telegraphic nuise type in the neutralization factors. Middle: Fluctuations in the equilibrium radius induced by these perturbations. Bottom: Corresponding phase space trajectory of an internal test particle. 


\section{Weakly colored noise}

In this section, the Stratonovich approach $[9,13]$ for the analysis of stochastic differential equations is used to analyze the case where the neutralization factors contain arbitrary fluctuations with short correlation times, i.e. weakly colored noise: from these results the effect of white noise perturbations is obtained as a particular case.

Stratonovich requires the starting set of equations to be in the following format:

$$
\frac{\mathrm{d} x}{\mathrm{~d} t}=\epsilon F[x, t ; \varphi],
$$

where $\boldsymbol{x}$ represents the state vector, $\epsilon$ is a smallness parameter used by Stratonovich as an expansion parameter and $\varphi$ is a random variable with probability density function $D(\varphi)$. Thus, given an initial condition $x_{0}$, different realizations of the process $\{x(t)\}$ are obtained for different outcomes of $\varphi$ [3]. The conditional probability density function $P\left(x, t \mid x_{10}, 0\right)$ is obtained from

$$
P\left(x, t \mid x_{0}, 0\right)=\int \mathrm{d} \varphi D(\varphi) \delta\left[x-x\left(t ; x_{0} \mid \varphi\right)\right],
$$

where $x\left(t ; x_{0} \mid \varphi\right)$ is the solution of eq. (13) given the initial condition $x_{0}$, for a given outcome of the random variable $\varphi$.

Starting from eqs. (13) and (14) the following Fokker-Planck like equation to be satisfied by $P\left(x, t \mid x_{0}, 0\right)$ [which will be abbreviated from now on to $P(x, t)]$. for times greater than the correlation times of the fluctuations, can be obtained, see appendix $A$ :

$$
\begin{aligned}
\frac{\partial}{\partial t} P(x, t)= & -\epsilon \frac{\partial}{\partial x_{t}}\left[\eta_{i}(x, t) P(x, t)\right] \\
& +\epsilon^{2} \frac{\partial^{2}}{\partial x_{\imath} \partial x_{j}}\left[P(x, t) \int_{0}^{x} \mathrm{~d} \tau K_{t \prime}(x, t ; x, \tau)\right] \\
& -\epsilon^{2} \frac{\partial}{\partial x_{i}}\left[P(x, t) \int_{u}^{x} \mathrm{~d} \tau\left(\frac{\partial}{\partial u_{,}} K_{t j}(u, t ; x, \tau)\right)_{u=x}\right]+C\left(\epsilon^{3}\right),
\end{aligned}
$$

where summation over repeated indices is understood; in the above equation we have used the following definitions:

$$
\eta_{t}(x, t) \equiv \int \mathrm{d} \varphi D(\varphi) F_{1}(x, t ; \varphi)
$$


for the mean, and

$$
K_{l \jmath}(u, t ; \boldsymbol{x}, \tau) \equiv \int \mathrm{d} \varphi D(\varphi) F_{l}(u, t ; \varphi) F_{,}(x, \tau ; \varphi)-\eta_{l}(u, t) \eta,(x, \tau)
$$

for the covariances of $F(x, t ; \varphi)$.

Analysis of the stability in the mean

In order to simplify the application of the Stratonovich technique, we make the transformation

$$
S(t)=\mathrm{e}^{-1 \Omega_{1,} 2 \gamma} Z(t),
$$

where $Z(t)$ is a complex function of time; after substitution in eq. (4) we obtain the following differential equation for $Z(t)$ :

$$
\frac{\mathrm{d}^{2} Z}{\mathrm{~d} t^{2}}+\omega_{0}^{2} Z(t)=0
$$

where $\omega_{0}^{2}$ has been defined in eq. (3); this equation represents the behavior of a classical harmonic oscillator. In the case where the neutralization factors contain a random term, it becomes the equation of a stochastic harmonic uscillator,

$$
\frac{\mathrm{d}^{2} Z}{\mathrm{~d} t^{2}}+\omega_{i 1}^{2}[1+\epsilon m(t ; \varphi)] Z(t)=0
$$

where $\epsilon$ is the smallness parameter needed in the Stratonovich theory; and $m(t ; \varphi)$ is a realization of the random process $\{m(t)\}$ generated given an outcome of the stochastic variable $\varphi$, which is assumed to have a probability density function $D(\varphi)$; in eq. (20) was assumed that $\omega_{0}^{2} \neq 0$. The random process $\{m(t)\}$ will be chosen to be a stationary stochastic process with the following properties:

$$
\langle m(t)\rangle=0, \quad\left\langle m(t) m\left(t_{0}\right)\right\rangle=C\left(\left|t-t_{0}\right|\right) .
$$

Eq. (20) is a second order differential equation which can be set as two coupled first order differential equations, and casted in the format required in eq. (13), by a change of variables, see appendix B; then a Fokker-Planck-like equation can be obtained, for the conditional probability density function $P(Z, \dot{Z}, t)$. Multiplying this equation by $Z$ and integrating over all possibles values of $Z$, and $\dot{Z}$, the following approximate equation for the mean value, 
$\langle Z(t)\rangle$, is derived:

$$
\begin{aligned}
& \frac{\mathrm{d}^{2}}{\mathrm{~d} t^{2}}\langle Z(t)\rangle+\epsilon^{2} \omega_{0}^{2} \int_{0}^{\pi} \mathrm{d} \tau C(\tau) \sin ^{2}\left(\omega_{0} \tau\right) \frac{\mathrm{d}}{\mathrm{d} t}\langle Z(t)\rangle \\
& \quad+\omega_{0}^{2}\left[1-\epsilon^{2} \frac{\omega_{0}}{2} \int_{0}^{x} \mathrm{~d} \tau C(\tau) \sin \left(2 \omega_{0} \tau\right)\right]\langle Z(t)\rangle=0
\end{aligned}
$$

where terms of the order $C\left(\epsilon^{3}\right)$ have been neglected.

This equation has been obtained before by other authors, using the Bourret approximation [7]. As pointed out by van Kampen [7], the effect of colored noise is to produce a shift in the frequency, as weli as a damping in the averaged oscillations. If the second term of eq. (22) is negative, this dampıng is negative and the amplitude of the oscillations will grow in time; this happens if the fluctuations are particularly strong at twice the frequency of the unperturbed oscillators, i.e. $2 \omega_{11}$; we shall point out that this value corresponds to the frequency of the radial oscillations of a test particle when $\omega_{0}^{2}$ is constant, as can be deduced from eq. (12).

Since the term $\exp \left\{-\mathrm{i} \Omega_{0} t / 2 \gamma\right\}$ in eq. (18) produces only periodic rotations, the stability of the test particle is determined only by the time behavior of $Z(t)$; thus, the mean position $\langle S(t)\rangle$ will be unstable if the magnitude of the power spectral density of the fluctuations evaluated at $2 \omega_{0}$ is larger than that at $\omega=0$.

\section{Mean square stability analysis}

In order to analyze the behavior of the mean square radius in the presence of random fluctuations in the neutralization factors, we use eq. (11). Assuming again that in the unperturbed case $\omega_{0}^{2} \neq 0$, when the neutralization factors suffer from random perturbations, we write again

$$
\omega_{0}^{2}(t)=\omega_{0}^{2}[1+\epsilon m(t ; \varphi)],
$$

where $m(t ; \varphi)$ is a realization of the random process $\{m(t)\}$, whose properties are specified in eq. (21). Thus, eq. (11) is written now as

$$
\frac{\mathrm{d}^{3}}{\mathrm{~d} t^{3}} R^{2}+4 \omega_{0,}^{2}[1+\epsilon m(t ; \varphi)] \frac{\mathrm{d}}{\mathrm{d} t} R^{2}+2 \omega_{10}^{2} \epsilon \dot{m}(t ; \varphi) R^{2}=0,
$$

where $\dot{m}(t ; \varphi)$ is the time derivative of $m(t ; \varphi)$.

The above equation for $R^{2}(t)$ is a third order differential equation which can be written as a set of three first order differential equations. Transforming this set into the standard form, eq. (13), as shown in appendix B, a Fokker- 
Planck-like equation of the form given in eq. (15) can be obtained; in this case the state variables are: $R^{2}, \mathrm{~d} R^{2} / \mathrm{d} t$, and $\mathrm{d}^{2} R^{2} / \mathrm{d} t^{2}$. Multiplying this equation by $R^{2}$ and integrating over all possible values of the statc variables, we obtain the following approximate equation for the mean square radius:

$$
\begin{gathered}
\frac{\mathrm{d}^{3}}{\mathrm{~d} t^{3}}\left\langle R^{2}\right\rangle+4 \omega_{0}^{2}\left[1-\epsilon^{2} \frac{\omega_{0}}{2} \int_{0}^{x} \mathrm{~d} \tau C(\tau) \sin \left(2 \omega_{0} \tau\right)\right] \frac{\mathrm{d}}{\mathrm{d} t}\left\langle R^{2}\right\rangle \\
-4 \omega_{0}^{4} \epsilon^{2} \int_{0}^{x} \mathrm{~d} \tau C(\tau) \cos \left(2 \omega_{0} \tau\right)\left\langle R^{2}(t)\right\rangle=0 .
\end{gathered}
$$

Since the last term in the above equation is proportional to the power spectral density evaluated at $2 \omega_{0}$, the last term is always non-positive. According to the Routh-Hurwitz criterion, a necessary condition for the solution of a differential equation to be bounded is that the coefficients of the different terms in the equation should have the same sign [14]. This is not the case in eq. (25) and the mean square radius is therefore unstable, even for small magnitude fluctuations, whenever its power spectral density contains a component of frequency $2 \omega_{0}$, the frequency of the radial oscillations in an unperturbed beam, as discussed in the introduction.

Therefore, even if the mean position of a test particle describes bounded trajectories in the $x-y$ plane, the dispersion around this mean position, determined by the behavior of the mean square radius, grows with time, and the beam is probabilistically unstable.

Although the exact solution of eq. (25) is available. if we define

$$
\omega^{2} \equiv \omega_{0}^{2}\left[1-\epsilon^{2} \frac{\omega_{0}}{2} \int_{0}^{x} \mathrm{~d} \tau C(\tau) \sin \left(2 \omega_{0} \tau\right)\right]
$$

and

$$
\xi \equiv \omega_{0}^{2} \epsilon^{2} \int_{0}^{x} \mathrm{~d} \tau C(\tau) \cos \left(2 \omega_{0} \tau\right),
$$

an approximate solution can be obtained when $\xi \ll \omega^{3} / 4 \omega_{i}^{2}$, which is given by

$$
\left\langle R^{2}(t)\right\rangle=A_{01} \mathrm{e}^{\omega_{10}^{2} \xi t / \omega^{2}}+A_{1} \mathrm{e}^{-\omega_{0}^{2} \xi t / 2 \omega^{2}} \sin (2 \omega t+\phi) ;
$$

this implies that the magnitude of the oscillations of the mean square radius decays in time, while its average value grows exponentially. We should notice that in the absence of fluctuations, $\epsilon=0$, eq. (27) reduces to eq. (12) which corresponds to a noiseless beam. 


\section{White noise fluctations}

The case of white noise perturbations can be obtained from the above results, when we substitute $C(\tau)=2 D \delta(\tau)$ for the correlation function. where $D$ is the strength of the fluctuations. It follows, from eq. (22), that the equation for the mean $\langle Z(t)\rangle$ reduces to

$$
\frac{\mathrm{d}^{2}\langle Z(t)\rangle}{\mathrm{d} t^{2}}+\omega_{0}^{2}\langle Z(t)\rangle=0
$$

which is identical to the noiseless equation, eq. (19); then, the mean will be stable if $\omega_{0}^{2}>0$. The equation for the mean square radius, eq. (25), reduces to

$$
\frac{\mathrm{d}^{3}}{\mathrm{~d} t^{3}}\left\langle R^{2}\right\rangle+4 \omega_{0}^{2} \frac{\mathrm{d}}{\mathrm{d} t}\left\langle R^{2}\right\rangle-4 \omega_{10}^{4} \epsilon^{2} D\left\langle R^{2}(t)\right\rangle=0 .
$$

When we have Gaussian white noise the above equation becomes exact. regardless the magnitude of $\epsilon$ since higher order terms vanish exactly, and we can set $\epsilon=1$. The solution of eq. (29) is always unstable in the presence of Gaussian white noise. If the strength of the fluctuations, $D$, satisfies $D \ll$ $1 / 4 \omega_{0}$, the time behavior of the mean square radius can be approximated by

$$
\left\langle R^{2}(t)\right\rangle=A_{0} \mathrm{e}^{\omega_{i j}^{j} D t}+A_{1} \mathrm{c}^{-\omega_{i 1}^{2} D t 2} \sin \left(2 \omega_{11} t+\phi\right) .
$$

From the above results we can point out some of the short-comings of the use of white noise. as compared with the weakly colored noise. In this problem, white noise predicts always a stable mean, in fact these equations are identical to those of a noiseless beam; while in the weakly colored noise case, the mean may or may not be stable. The colored noise produces a shift in the frequency of the oscillations of the mean position, as compared to a noiseless beam. The white noise yields always an unstable mean square radius; the weakly colored noise predicts instability only when the power spectral density of the perturbations contains a component at the frequency of the radial oscillations.

In the next section, we find the exact solution to the case in which the fluctuations are of the telegraphic noise type. In order to compare later on the approximate results derived from Stratonovich's theory with those obtained in the next section, we need the correlation function of a telegraphic noise that takes only the values $\{+\eta,-\eta\}$ with equal probability, as will be discussed in section 4 . The correlation function of this random process is $C(\tau)=$ $\eta^{2} \exp \{-2 \lambda|\tau|\}$, where $\lambda$ is the mean reversal rate. Substituting this correlation function in eqs. (22) and (25), we obtain that the mean position is governed by 
the equation

$$
\frac{\mathrm{d}^{2}}{\mathrm{~d} t^{2}}\langle Z\rangle+\frac{\epsilon^{2} \eta^{2}}{4 \lambda} \frac{\omega_{0}^{4}}{\lambda^{2}+\omega_{0}^{2}} \frac{\mathrm{d}}{\mathrm{d} t}\langle Z\rangle+\omega_{0}^{2}\left[1-\frac{\epsilon^{2} \eta^{2}}{4} \frac{\omega_{0}^{2}}{\lambda^{2}+\omega_{0}^{2}}\right]\langle Z\rangle=0,
$$

and the mean square radius is obtained from the solution of

$$
\frac{\mathrm{d}^{3}}{\mathrm{~d} t^{3}}\left\langle R^{2}\right\rangle+\left[4 \omega_{11}^{2}-\frac{\epsilon^{2} \omega_{0}^{4} \eta^{2}}{\lambda^{2}+\omega_{0}^{2}}\right] \frac{\mathrm{d}}{\mathrm{d} t}\left\langle R^{2}\right\rangle-\frac{2 \lambda \eta^{2} \omega_{0}^{4}}{\lambda^{2}+\omega_{0}^{2}} \epsilon^{2}\left\langle R^{2}\right\rangle=0
$$

Expanding eqs. (31) and (32) in powers of the dimensionless parameter $\omega_{11} / \lambda$, we get for the mean position

$$
\begin{aligned}
& \frac{\mathrm{d}^{2}}{\mathrm{~d} t^{2}}\langle Z\rangle+\frac{\epsilon^{2} \eta^{2}}{4 \lambda^{3}} \omega_{0}^{4}\left(1-\frac{\omega_{0}^{2}}{\lambda^{2}}+\cdots\right) \frac{\mathrm{d}}{\mathrm{d} t}\langle Z\rangle \\
& \quad+\omega_{0}^{2}\left[1-\frac{\epsilon^{2} \eta^{2} \omega_{0}^{2}}{4 \lambda^{2}}\left(1-\frac{\omega_{0}^{2}}{\lambda^{2}}+\cdots\right)\right]\langle Z\rangle=0,
\end{aligned}
$$

and for the mean square radius we have

$$
\begin{gathered}
\frac{\mathrm{d}^{3}}{\mathrm{~d} t^{3}}\left\langle R^{2}\right\rangle+\left[4 \omega_{11}^{2}-\frac{\epsilon^{2} \eta^{2} \omega_{11}^{4}}{\lambda^{2}}\left(1-\frac{\omega_{0}^{2}}{\lambda^{2}}+\cdots\right)\right] \frac{\mathrm{d}}{\mathrm{d} t}\left\langle R^{2}\right\rangle \\
-\frac{2 \epsilon^{2} \eta^{2} \omega_{01}^{4}}{\lambda}\left(1-\frac{\omega_{01}^{2}}{\lambda^{2}}+\cdots\right)\left\langle R^{2}\right\rangle=0 .
\end{gathered}
$$

In the case where $\omega_{0}^{2} / \lambda^{2} \ll 1$, the Routh-Hurwitz criterion predicts that the solution of eq. (33) will be stable if the magnitude, $\epsilon \eta$. of the fluctuations satisfies the following inequalities:

$$
\omega_{0}^{2}>0, \quad \epsilon \eta<\frac{2 \lambda}{\omega_{0}} ;
$$

the mean square radius obtained from eq. (34) is always unstable according to the Routh-Hurwitz criterion. The white noise case is recovered in eqs. (33) and (34) when $C(\tau)=\eta^{2} \exp (-2 \lambda|\tau|) \rightarrow 2 D \delta(\tau)$, i.e. when $\lim _{\lambda \rightarrow x} \eta^{2 / 2} \lambda=D$.

\section{Telegraphic noise, an exact result}

In this section, following a procedure by Akcasu and Hammouda [15], is obtained the exact solution to the case in which the fluctuations in the 
neutralization factors form a Markov process $\{n(t)\}$, whose member functions take only two values: $+\boldsymbol{\eta}$ or $-\eta$; and makes independent jumps between these values following a Poisson distribution $P(m, t)=(\lambda t)^{m} \exp (-\lambda t) / m$ !, where $m$ is the number of jumps in a time interval of duration $t$, and $\lambda$ is the mean number of jumps per unit time.

Taking eq. (4) as the equation of motion in the $S$-plane and making the transformation in eq. (18), we obtain eq. (19). Assuming that the neutralization factors contain a fluctuating term with properties that were described above, we write eq. (19) as

$$
\frac{\mathrm{d}^{2} Z}{\mathrm{~d} t}+\omega_{0}^{2}[1+n(t)] Z(t)=0
$$

the solution of this equation for a given realization $n(t)$ can be written as [16]

$$
Z(t)=Z_{0} X_{1}(t)+\dot{Z}_{0} X_{2}(t)
$$

where $X_{1}(t)$ and $X_{2}(t)$ are two real independent solutions of eq. (36), satisfying

$$
X_{1}(0)=1, \quad \dot{X}_{1}(0)=0, \quad X_{2}(0)=0, \quad \dot{X}_{2}(0)=1 .
$$

The mean behavior of the solution of eq. (4) is then

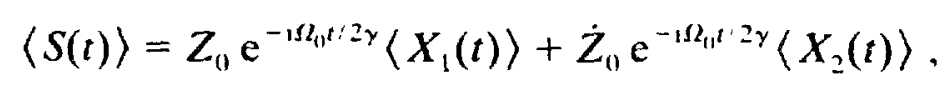

and the mean square radius is given by

$$
\left\langle|S(t)|^{2}\right\rangle=\left|Z_{0}\right|^{2}\left\langle X_{1}^{2}(t)\right\rangle+2 \operatorname{Re}\left(Z_{0}^{*} \dot{Z}_{0}\right)\left\langle X_{1}(t) X_{2}(t)\right\rangle+\left|\dot{Z}_{0}\right|^{2}\left\langle X_{2}^{2}(t)\right\rangle,
$$

where $\operatorname{Re}$ means "real part of", and $Z_{0}^{*}$ denotes the complex conjugate of $Z_{0}$. Notice that $Z_{0}=S(0)=x(0)+\mathrm{i} y(0)$ is the initial position, and $\dot{Z}_{0}$ is determined from the initial velocity, $\dot{S}(0)$, given by $v_{\mathrm{r}}(0)+\mathrm{i} v_{3}(0)=\dot{Z}_{01}-\mathrm{i} Z_{0} \Omega_{01} / 2 \gamma$.

Although eq. (36) is of second order, the solutions $X_{1}(t)$ and $X_{2}(t)$ together with their time derivatives do not constitute a Markov process, since in addition to the initial values of the above quanrities, we need the initial value of $n(t)$. Thus, we define a vector $\xi$ as

$$
\xi \equiv\left[X_{1}, \dot{X}_{1}, X_{2}, \dot{X}_{2}\right]^{\dagger},
$$

such that the process $\{\xi . n\}$ is Markov; this can be seen, since $n(t)$ and $\xi(t)$ are 
a consequence of the initial values $\xi(0), n(0)$, and the distribution and number of jumps in the time interval $[0, t]$, where, as already pointed out, the number of jumps in any time interval is independent of the number of jumps in another non-overlaping interval.

The state vector $\xi$ satisfies the equation

$$
\frac{\mathrm{d}}{\mathrm{d} t} \xi=M[n(t)] \xi,
$$

where

$$
M[n]=\left[\begin{array}{cccc}
0 & 1 & 0 & 0 \\
-\omega_{0}^{2}(1+n) & 0 & 0 & 0 \\
0 & 0 & 0 & 1 \\
0 & 0 & -\omega_{0}^{2}(1+n) & 0
\end{array}\right]
$$

The conditional probability density function $P\left(\xi, n, t \mid \xi_{1}, n_{0}, 0\right)$ of the Markov process $\{\xi, n\}$ satisfies the following Chapman-Kolmogorov equation:

$$
\begin{aligned}
& P\left(\xi, n, t+\Delta t \mid \xi_{0}, n_{0}, 0\right) \\
& \quad=\sum_{m--n}^{+n} \int \mathrm{d} \xi^{\prime} P\left(\xi, n, t+\Delta t \mid \xi^{\prime}, m, t\right) P\left(\xi^{\prime}, m, t \mid \xi_{0}, n_{0}, 0\right) \\
& \quad \text { for } t, \Delta t>0
\end{aligned}
$$

or

$$
\begin{aligned}
& P\left(\xi, n, t \mid \xi_{0}, n_{0}, 0\right) \\
& \quad=\sum_{m=-\eta}^{+\eta} \int \mathrm{d} \xi^{\prime} P\left(\xi, n, t \mid \xi^{\prime}, m, t-\Delta t\right) P\left(\xi^{\prime}, m, t-\Delta t \mid \xi_{1}, n_{0}, 0\right), \\
& \quad \text { for } t>\Delta t>0 .
\end{aligned}
$$

In order to derive a master equation, we need the following expression:

$$
\begin{aligned}
P\left(\xi, n, t \mid \xi^{\prime}, m, t-\Delta t\right)= & (1-\lambda \Delta t) \delta_{n, m} \delta\left(\xi+\delta \xi[\xi, n,-\Delta t]-\xi^{\prime}\right) \\
& +\lambda \Delta t \delta_{m,-n} \delta\left(\xi+\delta \xi[\xi, n,-\Delta t]-\xi^{\prime}\right) \\
& +C\left(\Delta t^{2}\right) .
\end{aligned}
$$

Substituting this expression in eq. (44b) and expanding,

$$
\begin{aligned}
& P\left(\xi-\delta \xi[\xi, n,-\Delta t], \pm n, t-\Delta t \mid \xi_{0}, n_{0}, 0\right) \\
& \quad=P\left(\xi, \pm n, t-\Delta t \mid \xi_{0}, n_{0}, 0\right)-\left[\frac{\partial P}{\partial \xi}\right]^{\dagger} \delta \xi[\xi, n,-\Delta t]+\cdots,
\end{aligned}
$$


where $\delta \xi[\xi, n,-\Delta t]$ is the increment in $\xi$ for a given value $n$ and an increment $-\Delta t$ in time; using eq. (42) to calculate this increment, the following master equation is obtained:

$$
\begin{aligned}
\frac{\partial}{\partial t} P\left(\xi, n, t \mid \xi_{0}, n_{0}, 0\right)= & -\frac{\partial P}{\partial \xi} M[n] \xi+\lambda\left[P\left(\xi,-n, t \mid \xi_{0}, n_{0}, 0\right)\right. \\
& \left.-P\left(\xi, n, t \mid \xi_{0}, n_{0}, 0\right)\right] .
\end{aligned}
$$

Now, defining

$$
P_{1}\left(\xi, t \mid \xi_{0}, n_{0}, 0\right) \equiv P\left(\xi,+\eta, t \mid \xi_{11}, n_{01}, 0\right)
$$

and

$$
P_{2}\left(\xi, t \mid \xi_{0}, n_{0}, 0\right) \equiv P\left(\xi,-\eta, t \mid \xi_{0}, n_{0}, 0\right)
$$

eq. (47) leads to the following set of coupled differential equations:

$$
\begin{aligned}
\frac{\partial P_{1}}{\partial t}= & -\dot{X}_{1} \frac{\partial P_{1}}{\partial X_{1}}+\omega_{01}^{2}(1+\eta) X_{1} \frac{\partial P_{1}}{\partial \dot{X}_{1}}-\dot{X}_{2} \frac{\partial P_{1}}{\partial X_{2}}+\omega_{0}^{2}(1+\eta) X_{2} \frac{\partial P_{1}}{\partial \dot{X}_{2}} \\
& +\lambda\left[P_{2}\left(\xi, t \mid \xi_{0}, n_{0}, 0\right)-P_{1}\left(\xi, t \mid \xi_{10}, n_{0}, 0\right)\right]
\end{aligned}
$$

and

$$
\begin{aligned}
\frac{\partial P_{2}}{\partial t}= & -\dot{X}_{1} \frac{\partial P_{2}}{\partial X_{1}}+\omega_{0}^{2}(1-\eta) X_{1} \frac{\partial P_{2}}{\partial \dot{X}_{1}}-\dot{X}_{2} \frac{\partial P_{2}}{\partial X_{2}}+\omega_{01}^{2}(1-\eta) X_{2} \frac{\partial P_{2}}{\partial \dot{X}_{2}} \\
& +\lambda\left[P_{1}\left(\xi, t \mid \xi_{0}, n_{0}, 0\right)-P_{2}\left(\xi, t \mid \xi_{0}, n_{0}, 0\right)\right] .
\end{aligned}
$$

which should be solved with the following initial conditions:

$$
\begin{aligned}
& P_{1}\left(\xi, 0 \mid \xi_{0}, n_{0}, 0\right)=\delta\left(\xi-\xi_{01}\right) \delta_{n_{01}+\eta}, \\
& P_{2}\left(\xi, 0 \mid \xi_{0}, n_{01}, 0\right)=\delta\left(\xi-\xi_{01}\right) \delta_{n_{11}-\eta} .
\end{aligned}
$$

Thus, given a function $\phi(\xi)$, we can use eq. (48) to calculate the quantity

$$
{ }_{n_{11}}\langle\phi\rangle_{i} \equiv \int \mathrm{d} \xi \phi(\xi) P_{i}\left(\xi, t \mid \xi_{01}, n_{0}, 0\right), \quad i=1,2 .
$$

which is the conditional average of the given function $\phi(\xi)$ at time $t$, given $n(0)=n_{0}$, and $\xi(0)=\xi_{0}$, where $n(t)=+\eta$. if $i=1$, or $n(t)=-\eta$, if $i=2$. 
Since the initial value $n_{0}$ in general cannot be determined, the conditional average at time $t$, of $\phi(\xi)$, given $\xi(0)=\xi_{0}$. must contain an average over the initial val'des $n_{0}$; also since we are not interested in a particular value of $n$ at the time $t$, we shall sum over the different values of $i$ in eq. (51). Therefore, if $\mathscr{P}\left(n_{0}\right)$ is the probability distribution of $n$ at time $t=0$, then

$$
\langle\phi\rangle=\sum_{i=1}^{2} \sum_{n_{11}--\eta}^{+\eta} \mathscr{P}\left(n_{11}\right)_{n_{01}}\langle\phi\rangle,
$$

yields the conditional average at time $t$ of $\phi(\xi)$ given $\xi(0)=\xi_{1}$.

Assuming that $n_{0}$ takes the values $(+\eta,-\eta)$ with equal probability, this is $\mathscr{P}\left(n_{0}\right)=\frac{1}{2} \delta_{n_{1},+\eta}+\frac{1}{2} \delta_{n_{11}, \eta}$, and defining

$$
\langle\phi(t)\rangle_{1} \equiv \frac{1}{2} \int \mathrm{d} \xi \phi(\xi)\left[P_{1}\left(\xi, t \mid \xi_{10},+\eta, 0\right)+P_{1}\left(\xi, t \mid \xi_{0,},-\eta, 0,\right]\right.
$$

and

$$
\langle\phi(t)\rangle_{2} \equiv \frac{1}{2} \int \mathrm{d} \xi \phi(\xi)\left[P_{2}\left(\xi, t \mid \xi_{0},+\eta, 0\right)+P_{2}\left(\xi, t \mid \xi_{1,},-\eta, 0\right)\right] .
$$

eq. (52) can be written as

$$
\langle\phi(t)\rangle=\langle\phi(t)\rangle_{1}+\langle\phi(t)\rangle_{2} .
$$

With the above results, the first order moments of eqs. (47) vield the following set of equations:

$$
\frac{\mathrm{d}}{\mathrm{d} t}\left[\begin{array}{c}
\left\langle X_{l}\right\rangle_{1} \\
\left\langle X_{l}\right\rangle_{2} \\
\left\langle\dot{X}_{l}\right\rangle_{1} \\
\left\langle\dot{X}_{t}\right\rangle_{2}
\end{array}\right]=\left[\begin{array}{cccc}
-\lambda & \lambda & 1 & 0 \\
\lambda & -\lambda & 0 & 1 \\
-\omega_{0}^{2}(1+\eta) & 0 & -\lambda & \lambda \\
0 & -\omega_{0}^{2}(1-\eta) & \lambda & -\lambda
\end{array}\right]\left[\begin{array}{c}
\left\langle X_{l}\right\rangle_{1} \\
\left\langle X_{l}\right\rangle_{2} \\
\left\langle\dot{X}_{l}\right\rangle_{1} \\
\left\langle\dot{X}_{l}\right\rangle_{2}
\end{array}\right]
$$

for $l=1.2$.

which have to be solved with the initial conditions

$$
\left[\begin{array}{c}
\left\langle X_{l}(0)\right\rangle_{1} \\
\left\langle X_{l}(0)\right\rangle_{2} \\
\left\langle\dot{X}_{l}(0)\right\rangle_{1} \\
\left\langle\dot{X}_{l}(0)\right\rangle_{2}
\end{array}\right]=\frac{1}{2}\left[\begin{array}{c}
\delta_{1} l \\
\delta_{1 l} \\
\delta_{2, l} \\
\delta_{2, l}
\end{array}\right], \quad l=1,2
$$

Since we are interested in $\left\langle X_{1}\right\rangle=\left\langle X_{1}\right\rangle_{1}+\left\langle X_{1}\right\rangle_{2}$ and $\left\langle X_{2}\right\rangle=\left\langle X_{2}\right\rangle_{1}+$ 
$\left\langle X_{2}\right\rangle_{2}$, which determine the behavior of the mean position according to eq. (39), we define $\left\langle K_{l}\right\rangle \equiv\left\langle X_{l}\right\rangle_{1}-\left\langle X_{l}\right\rangle_{2}$ and $\left\langle x_{l}\right\rangle \equiv\left\langle\dot{X}_{l}\right\rangle_{1}-\left\langle\dot{X}_{1}\right\rangle_{2}$ and transform eq̣s. (55) into

$$
\frac{\mathrm{d}}{\mathrm{d} t}\left[\begin{array}{c}
\left\langle X_{l}\right\rangle \\
\left\langle\dot{X}_{l}\right\rangle \\
\left\langle K_{l}\right\rangle \\
\left\langle\chi_{l}\right\rangle
\end{array}\right]=\left[\begin{array}{cccc}
0 & 1 & 0 & 0 \\
-\omega_{0}^{2} & 0 & -\eta \omega_{0}^{2} & 0 \\
0 & 0 & -2 \lambda & 1 \\
-\eta \omega_{0}^{2} & 0 & -\omega_{0}^{2} & -2 \lambda
\end{array}\right]\left[\begin{array}{c}
\left\langle X_{l}\right\rangle \\
\left\langle\dot{X}_{l}\right\rangle \\
\left\langle K_{l}\right\rangle \\
\left\langle X_{l}\right\rangle
\end{array}\right], \quad l=1.2,
$$

where the initial conditions are now

$$
\left[\begin{array}{c}
\left\langle X_{l}\right\rangle \\
\left\langle\dot{X}_{l}\right\rangle \\
\left\langle K_{l}\right\rangle \\
\left\langle\chi_{l}\right\rangle
\end{array}\right]_{t=0}=\left[\begin{array}{c}
\delta_{1 . l} \\
\delta_{2 . l} \\
0 \\
0
\end{array}\right], \quad l=1,2
$$

In a similar way, after taking second order moments in eqs. (49), the following equations that determine the behavior of $\left\langle X_{1}^{2}\right\rangle,\left\langle X_{2}^{2}\right\rangle$ and $\left\langle X_{1} X_{2}\right\rangle$ can be obtained:

$$
\begin{gathered}
\quad\left[\begin{array}{c}
\left\langle X_{l}^{2}\right\rangle \\
\left\langle X_{l} \dot{X}_{l}\right\rangle \\
\left\langle\dot{X}_{l}^{2}\right\rangle \\
\left\langle\alpha_{l}\right\rangle \\
\left\langle\beta_{l}\right\rangle \\
\left\langle\gamma_{l}\right\rangle
\end{array}\right]=\left[\begin{array}{cccccc}
0 & 2 & 0 & 0 & 0 & 0 \\
-\omega_{0}^{2} & 0 & 1 & -\eta \omega_{0}^{2} & 0 & 0 \\
0 & -2 \omega_{0}^{2} & 0 & 0 & -2 \eta \omega_{11}^{2} & 0 \\
0 & 0 & 0 & -2 \lambda & 2 & 0 \\
-\omega_{0}^{2} \eta & 0 & 0 & -\omega_{0}^{2} & -2 \lambda & 1 \\
0 & -2 \omega_{0}^{2} \eta & 0 & 0 & -2 \omega_{0}^{2} & -2 \lambda
\end{array}\right]\left[\begin{array}{c}
\left\langle X_{l}^{2}\right\rangle \\
\left\langle X_{l} \dot{X}_{l}\right\rangle \\
\left\langle\dot{X}_{l}^{2}\right\rangle \\
\left\langle\alpha_{l}\right\rangle \\
\left\langle\beta_{l}\right\rangle \\
\left\langle\gamma_{l}\right\rangle
\end{array}\right], \\
l=1,2,
\end{gathered}
$$

which have to be solved with the initial conditions

$$
\left[\begin{array}{c}
\left\langle X_{l}^{2}\right\rangle \\
\left\langle X_{l} \dot{X}_{l}\right\rangle \\
\left\langle\dot{X}_{l}^{2}\right\rangle \\
\left\langle\alpha_{l}\right\rangle \\
\left\langle\beta_{l}\right\rangle \\
\left\langle\gamma_{l}\right\rangle
\end{array}\right]_{t=0}=\left[\begin{array}{c}
\delta_{1 . l} \\
0 \\
\delta_{2, l} \\
0 \\
0 \\
0
\end{array}\right], \quad l=1,2 ;
$$

and 


$$
\begin{aligned}
& \frac{\mathrm{d}}{\mathrm{d} t}\left[\begin{array}{c}
\left\langle X_{1} X_{2}\right\rangle \\
\langle\Gamma\rangle \\
\left\langle\dot{X}_{1} \dot{X}_{2}\right\rangle \\
\left\langle\alpha_{3}\right\rangle \\
\left\langle\beta_{3}\right\rangle \\
\left\langle\gamma_{3}\right\rangle
\end{array}\right] \\
& =\left[\begin{array}{cccccc}
0 & 1 & 0 & 0 & 0 & 0 \\
-2 \omega_{0}^{2} & 0 & 2 & -2 \eta \omega_{0}^{2} & 0 & 0 \\
0 & -\omega_{0}^{2} & 0 & 0 & -\eta \omega_{0}^{2} & 0 \\
0 & 0 & 0 & -2 \lambda & 1 & 0 \\
-2 \omega_{0}^{2} \eta & 0 & 0 & -2 \omega_{0}^{2} & -2 \lambda & 2 \\
0 & -\omega_{0}^{2} \eta & 0 & 0 & -\omega_{0}^{2} & -2 \lambda
\end{array}\right]\left[\begin{array}{c}
\left\langle X_{1} X_{2}\right\rangle \\
\langle\Gamma\rangle^{2} \\
\left\langle\dot{X}_{2} \dot{X}_{2}\right\rangle \\
\left\langle\alpha_{3}\right\rangle \\
\left\langle\beta_{3}\right\rangle \\
\left\langle\gamma_{3}\right\rangle
\end{array}\right],
\end{aligned}
$$

which have to be solved with the initial conditions

$$
\left[\begin{array}{c}
\left\langle X_{1} X_{2}\right\rangle \\
\langle\Gamma\rangle \\
\left\langle\dot{X}_{1} \dot{X}_{2}\right\rangle \\
\left\langle\alpha_{3}\right\rangle \\
\left\langle\beta_{3}\right\rangle \\
\left\langle\gamma_{3}\right\rangle
\end{array}\right]_{t-0}=\left[\begin{array}{l}
0 \\
1 \\
0 \\
0 \\
0 \\
0
\end{array}\right] ;
$$

in the above equations $\left\langle\alpha_{l}\right\rangle \equiv\left\langle X_{l}^{2}\right\rangle_{1}-\left\langle X_{l}^{2}\right\rangle_{2} ;\left\langle\beta_{l}\right\rangle \equiv\left\langle X_{l} \dot{X}_{l}\right\rangle_{1}-\left\langle X_{l} X_{l}\right\rangle_{2}$; $\left\langle\gamma_{1}\right\rangle \equiv\left\langle\dot{X}_{l}^{2}\right\rangle_{1}-\left\langle\dot{X}_{l}^{2}\right\rangle_{2} \quad$ for $l=1.2 ; \quad\left\langle\alpha_{3}\right\rangle \equiv\left\langle X_{1} X_{2}\right\rangle_{1}-\left\langle X_{1} X_{2}\right\rangle_{2}:\left\langle\beta_{3}\right\rangle \equiv$ $\left\langle\dot{X}_{1} X_{2}\right\rangle_{1}-\left\langle\dot{X}_{1} X_{2}\right\rangle_{2}+\left\langle X_{1} \dot{X}_{2}\right\rangle_{1}-\left\langle X_{1} \dot{X}_{2}\right\rangle_{2}:\left\langle\gamma_{3}\right\rangle \equiv\left\langle\dot{X}_{1} \dot{X}_{2}\right\rangle_{1}-\left\langle X_{1} X_{2}\right\rangle_{2}:$ and $\langle\Gamma\rangle \equiv\left\langle\dot{X}_{1} X_{2}\right\rangle+\left\langle\dot{X}_{1} \dot{X}_{2}\right\rangle$.

We should point out here that eqs. (57)-(62) reduce to the case of a noise free beam, when $\boldsymbol{\eta}=0$. In what follows, we shall use these equations and study the stability of their solutions.

\section{Analysis of the stability in the mean}

The behavior of the mean position of test particles is given in eq. (39): in this expression the factor $\exp \left(-\mathrm{i} \Omega_{0} t / 2 \gamma\right)$ represents only a rotational motion in the $S$-plane, thus, the stability in the mean is determined only by the behavior of $\left\langle X_{1}(t)\right\rangle$ and $\left\langle X_{2}(t)\right\rangle$. Taking the Laplace transform of eq. (57), the following characteristic equation is obtained:

$$
s^{4}+4 \lambda s^{3}+\left(4 \lambda^{2}+2 \omega_{0}^{2}\right) s^{2}+4 \lambda \omega_{0}^{2} s+4 \lambda^{2} \omega_{01}^{2}+\omega_{0}^{4}\left(1-\eta^{2}\right)=0 .
$$

Applying the Routh-Hurwitz criterion [14] to the above equation, the 
necessary and sufficient conditions for stability of the solution of eq. (57) are

$$
\omega_{0}^{2}>0, \quad \eta^{2}<1+4 \lambda^{2} \% \omega_{0}^{2} .
$$

The first requirement is the neutral stability condition of a noiseless beam as discussed before. The second condition establishes a maximum value for the magnitude of the fluctuations, if the oscillations are to be damped.

We should point out that conditions in eq. (64) reduces to the conditions in eq. (35) in the approximation of short correlation time, i.e. when $\lambda / \omega_{0} \gg 1$ and $\eta$ is replaced by $\epsilon \eta$.

\section{Mean square stability analysis}

It is easily checked that eqs. (59) and (61) lead to the same characteristic equation. which is

$$
\begin{aligned}
s^{6} & +6 \lambda s^{5}+4\left(2 \omega_{0}^{2}+3 \lambda^{2}\right) s^{4}+8 \lambda\left(4 \omega_{0}^{2}+\lambda^{2}\right) s^{3}+16 \omega_{0}^{2}\left[3 \lambda^{2}+\omega_{0}^{2}\left(1-\eta^{2}\right)\right] s^{2} \\
& +32 \lambda \omega_{0}^{2}\left[\lambda^{2}+\omega_{0}^{2}\left(1-\eta^{2}\right)\right] s-16 \eta^{2} \omega_{0}^{4} \lambda^{2}=0 .
\end{aligned}
$$

According to the Routh-Hurwitz criterion, if the roots of the characteristic equation do not have positive real parts is necessary that all the coefficients of the polynomial have the same sign and none of the coefficient vanishes. Since the last term in eq. (65) is always negative, then the solution of eqs. (59) and (61) and therefore the mean square radius will grow in time, regardless of the magnitude of the fluctuations. The beam is then probabilistically unstable.

\section{Comparison between the exact and Stratonovich results}

In what follows we shall compare eq. (33). which was obtained using Stratonovich's method in section (3) for the particular case of the telegraphist signal, with the exact equations for the mean position, eqs. (57), iollowing an expansion technique. Similarly, eq. (34) for the mean square radius obtained from the Stratonovich approach can be compared with the exact equations (59) and (61), following the same technique, but only the results will be shown here.

A) Equation for the mean. In the case of telegraphic noise, the mean position is given by eq. (39) where $\left\langle X_{1}(t)\right\rangle$ and $\left\langle X_{2}(t)\right\rangle$ are obtained from eq. (57) with initial conditions (58). We notice first that eq. (57) can be written as two coupled dif ${ }^{5}$ erential equations, i.e

$$
\frac{\mathrm{d}^{2}}{\mathrm{~d} t^{2}}\left\langle X_{l}\right\rangle+\omega_{0}^{2}\left\langle X_{l}\right\rangle=-\eta \omega_{0}^{2}\left\langle K_{l}\right\rangle
$$


and

$$
\frac{\mathrm{d}^{2}}{\mathrm{~d} t^{2}}\left\langle K_{l}\right\rangle+4 \lambda \frac{\mathrm{d}}{\mathrm{d} t}\left\langle K_{l}\right\rangle+\left(\omega_{0}^{2}+4 \lambda^{2}\right)\langle K .\rangle=-\eta \omega_{0}^{2}\left\langle X_{l}\right\rangle \quad \text { for } l=1,2
$$

which have to be solved with the following initial conditions:

$$
\begin{aligned}
& \left\langle X_{l}(0)\right\rangle=\delta_{1, l}, \quad \frac{\mathrm{d}}{\mathrm{d} t}\left\langle X_{l}(0)\right\rangle=\delta_{2, l}, \quad\left\langle K_{l}(0)\right\rangle=0, \quad \frac{\mathrm{d}}{\mathrm{d} t}\left\langle K_{l}(0)\right\rangle=0 \\
& \text { for } l=1,2 .
\end{aligned}
$$

Noticing that when $\eta=0$ eqs. (66) and (67) become decoupled, we look for a solution of the form

$$
\left\langle X_{l}(t)\right\rangle=Y_{l, 0}(t)+\eta Y_{l, 1}(t)+\eta^{2} Y_{l, 2}(t)+\cdots
$$

and

$$
\left\langle K_{l}(t)\right\rangle=\xi_{l, 0}(t)+\eta \xi_{l, 1}(t)+\eta^{2} \xi_{l, 2}(t)+\cdots
$$

Substituting the above expansions in eqs. (66) and (67) and equating the coefficients of equal powers of $\eta$, we obtain the following set of hierarchical equations:

$$
\begin{aligned}
& \frac{\mathrm{d}^{2}}{\mathrm{~d} t^{2}} Y_{l, m}+\omega_{0}^{2} Y_{l, m}=-\omega_{0}^{2}\left(1-\delta_{m .0}\right) \xi_{l, m-1} \quad \text { for } m=0,1,2, \ldots \\
& \frac{\mathrm{d}^{2}}{\mathrm{~d} t^{2}} \xi_{l, m}+4 \lambda \frac{\mathrm{d}}{\mathrm{d} t} \xi_{l, m}+\left(\omega_{0}^{2}+4 \lambda^{2}\right) \xi_{l, m}=-\omega_{0}^{2}\left(1-\delta_{m, 0}\right) Y_{l, m-1} \\
& \quad \text { for } m=0,1,2, \ldots ;
\end{aligned}
$$

this set of equations should be solved with the initial conditions

$$
Y_{l, m}(0)=\delta_{1, l} \delta_{m, 0}, \quad \dot{Y}_{l m}(0)=\delta_{2, l} \delta_{m 0}, \quad \xi_{l m}(0)=0, \quad \dot{\xi}_{l m}(0)=0 .
$$

It is easily seen that using the above initial conditions eqs. (69) and (70) take the form

$$
\left\langle X_{l}(t)\right\rangle=Y_{l .0}(t)+\eta^{2} Y_{l .2}(t)+\eta^{4} Y_{l, 4}(t)+\cdots \quad \text { for } l=1,2
$$


and

$$
\left\langle K_{l}(t)\right\rangle=\eta \xi_{l, 1}(t)+\eta^{3} \xi_{l, 3}(t)+\eta^{\varsigma} \xi_{l, 5}(t)+\cdots \quad \text { for } l=1,2 .
$$

The purpose now is to obtain an approximate expression for the solution $\xi_{l, m}(t)$ of eq. (72), in terms of $Y_{l, m-1}(t)$, assuming that the latter is a known function. With this in mind, we divide eq. (72) by $\left(\omega_{0}^{2}+4 \lambda^{2}\right)$ and expand the resulting equation in powers of $\left(\omega_{01} / \lambda\right)$. After that, we propose that the solution of this equation, $\xi_{l, m}(t)$, is written as an expansion in powers of $\left(\omega_{1} / \lambda\right)$, which is

$$
\xi_{l, m}(t)=\varphi_{l, m 0}(t)+\left(\frac{\omega_{0}}{\lambda}\right) \varphi_{l, m 1}(t)+\left(\frac{\omega_{0}}{\lambda}\right)^{2} \varphi_{l, m 2}(t)+\cdots
$$

Equating now the coefficients of equal powers of $\omega_{0} / \lambda$, we obtain the coefficients $\left\{\varphi_{l . m}, i=1,2, \ldots\right\}$ in terms of $Y_{l, m-1}(t)$ and its derivatives; substituting these coefficients in eq. (76), we have up to $n$ ter $\left(\omega_{11}^{6} / \lambda^{6}\right)$

$$
\begin{aligned}
\xi_{l, m}(t)= & \frac{1}{8 \omega_{0}^{3}}\left(\frac{\omega_{0}}{\lambda}\right)^{5} \frac{\mathrm{d}^{3}}{\mathrm{~d} t^{3}} Y_{l, m-1}-\frac{3}{16 \omega_{0}^{2}}\left(\frac{\omega_{0}}{\lambda}\right)^{4} \frac{\mathrm{d}^{2}}{\mathrm{~d} t^{2}} Y_{l, m-1} \\
& +\frac{1}{4 \omega_{0}}\left[\left(\frac{\omega_{0}}{\lambda}\right)^{3}-\frac{1}{2}\left(\frac{\omega_{0}}{\lambda}\right)^{5}\right] \frac{\mathrm{d}}{\mathrm{d} t} Y_{l, m-1} \\
& -\frac{1}{4}\left[\left(\frac{\omega_{01}}{\lambda}\right)^{2}-\frac{1}{4}\left(\frac{\omega_{01}}{\lambda}\right)^{4}\right] Y_{l, m-1}+C\left(\omega_{0}^{0} / \lambda^{0}\right) .
\end{aligned}
$$

Substituting this expresion in eq. (71) the following equation is obtained:

$$
\begin{aligned}
\frac{\mathrm{d}^{2}}{\mathrm{~d} t^{2}} Y_{l, m}+\omega_{0}^{2} Y_{l, m}= & -\frac{1}{8 \omega_{0}}\left(\frac{\omega_{0}}{\lambda}\right)^{5} \frac{\mathrm{d}^{3}}{\mathrm{~d} t^{3}} Y_{l, m-2}+\frac{3}{16}\left(\frac{\omega_{0}}{\lambda}\right)^{4} \frac{\mathrm{d}^{2}}{\mathrm{~d} t^{2}} Y_{l, m-2} \\
& -\frac{\omega_{0}}{4}\left[\left(\frac{\omega_{0}}{\lambda}\right)^{3}-\frac{1}{2}\left(\frac{\omega_{01}}{\lambda}\right)^{5}\right] \frac{\mathrm{d}}{\mathrm{d} t} Y_{l, m-2} \\
& +\frac{\omega_{0}^{2}}{4}\left[\left(\frac{\omega_{0}}{\lambda}\right)^{2}-\frac{1}{4}\left(\frac{\omega_{0}}{\lambda}\right)^{4}\right] Y_{l m-2} \\
& \left.+\mathcal{C}\left(\omega_{0}^{\mathrm{h}} / \lambda^{\prime \prime}\right)\right\}\left(1-\delta_{m, 11}\right)
\end{aligned}
$$

for $l=1,2$ and $m=0,2,4,6, \ldots$

Multiplying the above equation by $\eta^{m}$, adding the sequence of resulting equations, i.e. for $m=0,2 \ldots$, and recalling eq. (74) we obtain up to order 
$\omega_{11}^{6} / \lambda^{h}$ an approximate equation for $\left\langle X_{t}(t)\right\rangle$, which $\therefore$

$$
\begin{aligned}
& \frac{\eta^{2}}{8 \omega_{11}}\left(\frac{\omega_{11}}{\lambda}\right)^{5} \frac{\mathrm{d}^{3}}{\mathrm{~d} t^{3}}\left\langle X_{1}\right\rangle+\left[1-\frac{3 \eta^{2}}{16}\left(\frac{\omega_{11}}{\lambda}\right)^{4}\right] \frac{\mathrm{d}^{2}}{\mathrm{~d} t^{2}}\left\langle X_{t}\right\rangle \\
& +\eta^{2} \frac{\omega_{0}}{4}\left[\left(\frac{\omega_{11}}{\lambda}\right)^{3}-\frac{1}{2}\left(\frac{\omega_{01}}{\lambda}\right)^{5}\right] \frac{\mathrm{d}}{\mathrm{d} t}\left\langle X_{1}\right\rangle \\
& +\left\{\omega_{11}^{2}-\eta^{2} \frac{\omega_{01}^{2}}{4}\left[\left(\frac{\omega_{11}}{\lambda}\right)^{2}-\frac{1}{4}\left(\frac{\omega_{01}}{\lambda}\right)^{4}\right]\right\}\left\langle X_{l}(t)\right\rangle+\left(\left(\omega_{11}^{11} / \lambda^{n}\right)=0 .\right.
\end{aligned}
$$

for $l=1.2$.

Eq. (34) for the mean square radius in section 3 can be obtained following the same procedure. starting from the exact equations (59) or (61): this: however. will not be done here, only the results will presented.

B) Equation for the mean square radius. It can be shown that eqs (59) and (61) lead to the same set of coupled differential equations. the difference lics only in the initial conditions necessary to solve the equations; choosing eqs. (59) this set is

$$
\begin{aligned}
& \frac{\mathrm{d}^{3}}{\mathrm{~d} t^{3}}\left\langle X_{l}^{2}\right\rangle+4 \omega_{11}^{2} \frac{\mathrm{d}}{\mathrm{d} t}\left\langle X_{l}^{2}\right\rangle=-4 \eta \omega_{11}^{2} \frac{\mathrm{d}}{\mathrm{d} t}\left\langle\alpha_{l}\right\rangle-4 \eta \lambda \omega_{10}^{2}\left\langle\alpha_{l}\right\rangle . \\
& \frac{\mathrm{d}^{3}}{\mathrm{~d} t^{2}}\left\langle\alpha_{l}\right\rangle+6 \lambda \frac{\mathrm{d}^{2}}{\mathrm{~d} t^{2}}\left\langle\alpha_{l}\right\rangle+4\left[\omega_{11}^{2}+3 \lambda^{2}\right] \frac{\mathrm{d}}{\mathrm{d} t}\left\langle\alpha_{l}\right\rangle+8 \lambda\left(\omega_{11}^{2}+\lambda^{2}\right)\left\langle\alpha_{l}\right\rangle \\
& \quad=-4 \eta \omega_{11}^{2} \frac{\mathrm{d}}{\mathrm{d} t}\left\langle X_{l}^{2}\right\rangle-4 \eta \lambda \omega_{11}^{2}\left\langle X_{l}^{2}\right\rangle .
\end{aligned}
$$

which needs to be solved with the initial conditions

$$
\begin{array}{lll}
\left\langle X_{l}^{2}(0)\right\rangle=\delta_{l, 1} . & \frac{\mathrm{d}}{\mathrm{d} t}\left\langle X_{l}^{2}(0)\right\rangle=0 . & \frac{\mathrm{d}^{2}}{\mathrm{~d} t^{2}}\left\langle X_{l}^{2}(0)\right\rangle=-2 \omega_{11}^{2} \delta_{t .1}+2 \delta_{l .2} . \\
\left\langle\alpha_{l}\right\rangle=0 . & \frac{\mathrm{d}}{\mathrm{d} t}\left\langle\alpha_{l}\right\rangle=0 . & \frac{\mathrm{d}^{2}}{\mathrm{~d}^{2}}\left\langle\alpha_{l}\right\rangle=-2 \omega_{l 1}^{2} \eta \delta_{l .1} .
\end{array}
$$

Following a similar expansion technique to that used above. We find that $\left\langle X_{l}^{2}(t)\right\rangle$ satisfies

$$
-\frac{\eta^{2}}{8 \omega_{11}}\left(\frac{\omega_{01}}{\lambda}\right)^{5} \frac{\mathrm{d}^{4}}{\mathrm{~d} t^{4}}\left\langle X_{i}^{2}\right\rangle+\frac{\mathrm{d}^{3}}{\mathrm{~d} t^{3}}\left\langle X_{l}^{2}\right\rangle+\omega_{11}^{2} \eta^{2}\left[\left(\frac{\omega_{01}}{\lambda}\right)^{3}-\frac{1}{2}\left(\frac{\omega_{0}}{\lambda}\right)^{5}\right] \frac{\mathrm{d}^{2}}{\mathrm{~d} t^{2}}\left\langle X_{l}^{2}\right\rangle
$$




$$
\begin{aligned}
& +\left\{4 \omega_{0}^{2}-\eta^{2} \omega_{0}^{2}\left[\left(\frac{\omega_{0}}{\lambda}\right)^{2}+\left(\frac{\omega_{0}}{\lambda}\right)^{4}\right]\right\} \frac{\mathrm{d}}{\mathrm{d} t}\left\langle X_{l}^{2}\right\rangle \\
& -2 \omega_{0}^{3} \eta^{2}\left[\left(\frac{\omega_{0}}{\lambda}\right)-\left(\frac{\omega_{0}}{\lambda}\right)^{3}+\left(\frac{\omega_{0}}{\lambda}\right)^{5}\right]\left\langle X_{l}^{2}\right\rangle+\mathcal{O}\left(\omega_{0}^{6} / \lambda^{6}\right)=0 .
\end{aligned}
$$

When we compare the equations that describe the behavior of the mean position, eqs. (33) and (79), it follows that Stratonovich's result is correct, if terms of the order $\left(\omega_{0} / \lambda\right)^{4}$ and higher are neglected. Similarly, comparing the equations for the mean square radius, eqs. (34) and (83), it can be seen that Stratonovich's equation, eq. (34), is correct, if terms of the order $\left(\omega_{0} / \lambda\right)^{3}$ and higher can be ignored.

\section{Conclusions}

A test particle approach was used to study the stability of a simplified model of a charged particle beam subject to stochastic fluctuations in the neutralization factors.

The effects of weakly colored and telegraphic noise on the system were studied, and the stability was determined using the Routh-Hurwitz criterion.

The effect of weakly colored noise was studied using the Stratonovich approach for stochastic differential equations. It is found that the mean position of the test particle will be unstable, if the magnitude of the power spectral density of the fluctuations evaluated at the frequency of the radial osciliations of the particles in an unperturbed beam is larger than its magnitude at zero frequency. In addition, the beam will be probabilistically unstable whenever the power spectral density of the perturbations contain a component at frequency $2 \omega_{0}$, since in this case the mean square radius of the test particle is always an increasing function of time. The effect of white noise perturbations was obtained as a particular case of the weakly colored noise.

Finally, the exact solution for the case of telegraphic noise was obtained by means of the master equation of the process. It is found that the mean position of the test particle will be stable if the magnitude of the fluctuations satisfies certain constraints and if the unperturbed beam is neutrally stable. The mean square radius is always unsiabie regardless of the magnitude of the fiuctuations. These exact solutions are compared with those obtained from the weakly colored case using an expansion technique; it is concluded that Stratonovich's results are correct when terms of the order $\left(\omega_{0} / \lambda\right)^{4}$ and higher are ignored in the equation for the mean. and when terms of the order $\left(\omega_{11} / \lambda\right)^{3}$ and higher can be ignored in the equation for the mean square radius. However, when terms 
up to order $\left(\omega_{11} / \lambda\right)^{5}$ are included, the equation for the mean is a third order differential equation and the equation for the mean square a fourth order differential equation.

The conclusion is that within the limits of the model adopted in this work a relativistic charged particle beam is always unstable when the power spectral density of the fluctuations contains a component at the frequency of the radial oscillations of a test particle in an unperturbed beam.

\section{Acknowledgement}

The first author (J.V.E.) is indebted to Prof. A.Z. Akcasu for his invitation and warm hospitality during his visit to the Nuclear Enginecring Department of The University of Michigan. where most of this research was done.

\section{Appendix A}

In this appendix we present a derivation of the Fokker-Planck like equation. eq. (15), following closely refs. [9] and [13].

We start with the following stochastic differential equation:

$$
\frac{\mathrm{d} x}{\mathrm{~d} t}=\epsilon F(x, t: \varphi)
$$

where $\boldsymbol{x}(t)$ is an $N$-dimension state vector, $\epsilon$ is a smallness parameter, and $\varphi$ is a random variable with probability density function $D(\varphi)$. Given an initial condition $x_{10}$, different realizations of the process $\{x(t)\}$ will be obtained from the different outcomes $\varphi$. Therefore, the conditional probability density function $P\left(x, t \mid x_{11}, 0\right)$ is obtained from

$$
P\left(x, t \mid x_{11}, 0\right)=\int \mathrm{d} \varphi D(\varphi) \delta\left[x-x\left(t ; x_{11} \mid \varphi\right)\right]
$$

where $(x, t)$ are independent variabies and $x\left(t ; x_{1} \mid \bar{t}\right)$ denotes a particular realization of the process $\{x(t)\}$ for the initial condition $x\left(t=(1)=x_{11}\right.$. From now on. to shorten the notation, we shall write $P(x, t)$ instead of $P\left(x, t_{1}, x_{n}, 1\right)$. Defining

$$
Z\left(t, x_{01} \mid \varphi\right) \equiv x\left(t ; x_{11} \mid \varphi\right)-x_{11}
$$


and using the shift operator, we can write the delta function as

$$
\delta\left[x-x\left(t ; x_{0} \mid \varphi\right)\right]=\left[\mathrm{e}^{-Z_{t}\left(t, x_{0} \mid \varphi\right) d / \partial v_{i}} \delta(y)\right]_{y=x-x_{0}},
$$

where summation over repeated indices is understood; expanding the shift operator and substituting in (A.2) we obtain the following expression:

$$
P(x, t)=P(x, 0)+\hat{L} P(x, 0),
$$

where we have used $P(x, 0)=\delta\left(x-x_{0}\right)$ and defined the operator $\hat{L}$ as

$$
\begin{aligned}
\hat{L}(*) \equiv & \sum_{m_{1}=0}^{\infty} \cdots \sum_{m_{N}=0}^{\infty} \frac{(-1)^{m_{1}+\cdots+m_{N}}}{m_{1} ! \cdots m_{N} !} \\
& \times \frac{\partial^{m_{1}+}+m_{N}}{\partial x_{1}^{m_{1}} \cdots \partial x_{N}^{m_{N}}}\left[\overline{\left.Z_{1}^{m_{1}}(t ; \boldsymbol{x}) \cdots Z_{N}^{m_{N}(t ; x)} *\right] ;}\right.
\end{aligned}
$$

here the primes, ', in the summations are used to indicate that $m_{1}=m_{2}=$ $\ldots=m_{N}=0$ cannot be taken simultaneously; in eq. (A.6) we used the definition

$$
\overline{Z_{1}^{m_{1}}(t ; \boldsymbol{x}) \ldots Z_{N}^{m_{N}}(t ; \boldsymbol{x})} \equiv \int \mathrm{d} \varphi D(\varphi) Z_{1}^{m_{1}}(t ; x \mid \varphi) \cdots Z_{N}^{m_{N}}(t ; x \mid \varphi) .
$$

From eq. (A.5) we can write formally

$$
P(x, 0)=[1+\hat{L}]^{-1} P(x, t) ;
$$

taking partial derivatives with respect to time in eq. (A.5), and using eq. (A.8) we get

$$
\frac{\partial P(x, t)}{\partial t}=\frac{\partial \hat{L}}{\partial t}[1+\hat{L}]^{-1} P(x, t) .
$$

Now since $Z\left(t ; x_{0} \mid \varphi\right)$ satisfies the equation

$$
\frac{\mathrm{d}}{\mathrm{d} t} Z\left(t ; x_{0} \mid \varphi\right)=\epsilon F\left(Z+x_{0}, t ; \varphi\right),
$$

we expand $Z$ in powers of $\epsilon$, as

$$
Z(t ; x \mid \varphi)=\sum_{t=0}^{x} \epsilon^{\prime} Z_{(l)}(t ; x \mid \varphi)
$$


Substituting this expansion in eq. (A.10), and equating powers of $\epsilon$, we have

$$
\begin{aligned}
& Z_{(0)}(t ; x \mid \varphi)=0, \\
& Z_{(1)}(t ; x \mid \varphi)=\int_{0}^{t} \mathrm{~d} \tau F(x, \tau \mid \varphi), \\
& Z_{(2)}(t ; x \mid \varphi)=\int_{0}^{t} \mathrm{~d} \tau Z_{(1),}(\tau ; x \mid \varphi)\left(\frac{\partial}{\partial u_{u}} F(u, \tau \mid \varphi)\right)_{u=x},
\end{aligned}
$$

and so on, where we have used $Z(0 ; x \mid \varphi)=0$. Substituting eqs. (A.12) in (A.11) keeping only terms up to order $\epsilon^{2}$, and replacing the resulting expression in eq. (A.6), we find that the operator $\dot{L}$ can be written as

$$
\hat{L}=-\epsilon \hat{L}_{1}-\epsilon^{2} \hat{L}_{2}+\mathcal{O}\left(\epsilon^{3}\right)
$$

where

$$
\hat{L}_{1}(*)=\frac{\partial}{\partial x_{1}}\left[\overline{Z_{111}(t ; x)} *\right]
$$

and

$$
\hat{L}_{2}(*)=\frac{\dot{\partial}}{\partial x_{1}}\left[\overline{Z_{(2),}(t: x)} *\right]-\frac{1}{2} \frac{\partial^{2}}{\partial x_{1} \partial x_{1}}\left[\overline{Z_{(1),}(t ; x) Z_{(11,}(t: x)} *\right]
$$

Substituting eq. (A.13) in (A.9) and expanding in powers of $\epsilon$, we have

$$
\frac{\partial \hat{L}}{\partial t}(1+\hat{L})^{-1} P=-\epsilon \frac{\partial \hat{L}_{1}}{\partial t} P-\epsilon^{2}\left[\frac{\partial \hat{L}_{2}}{\partial t} P+\frac{\partial \hat{L}_{1}}{\partial t} \hat{L}_{1} P\right]+C\left(\epsilon^{3}\right),
$$

where, from eqs. (A.14) and (A.15), using eqs. (A.12), we have

$$
\begin{aligned}
\frac{\partial \hat{L}_{1}}{\partial t} P(x, t)= & \frac{\partial}{\partial x_{i}}\left[\overline{F_{t}(x, t)} P(x, t)\right], \\
\frac{\partial \hat{L}_{2}}{\partial t} P(x, t)= & \frac{\partial}{\partial x_{t}}\left[\int_{0}^{t} \mathrm{~d} \tau\left(\frac{\partial}{\partial u} \overline{F_{i}(u, t) F_{i}(x, \tau)}\right)_{u=x} P(x, t)\right] \\
& -\frac{\partial^{2}}{\partial x_{t} \partial x}\left[\int_{0}^{t} \mathrm{~d} \tau \overline{F_{2}(x, t) F_{i}(x, \tau)} P(x, t)\right]
\end{aligned}
$$


and

$$
\begin{aligned}
\frac{\partial \hat{L}_{1}}{\partial t} \hat{L}_{1} P(x, t)= & \frac{\partial^{2}}{\partial x_{t} \partial x_{t}}\left\{\int_{0}^{t} \mathrm{~d} \tau \overline{F_{t}(x, t)} \overline{F,(x, \tau)} P(x, t)\right\} \\
& -\frac{\partial}{\partial x_{t}}\left\{\int_{0}^{t} \mathrm{~d} \tau\left[\frac{\partial}{\partial u_{t}} \overline{F_{t}(u, t)} \overline{F_{,}(x, \tau)}\right]_{u=x} P(x, t)\right\} .
\end{aligned}
$$

Replacing eqs. (A.17)-(A.19) in eq. (A.16) and then the resulting expression in eq. (A.9), we get the fnllowing Fokker-Planck-like equation:

$$
\begin{aligned}
\frac{\partial}{\partial t} P(x, t)= & -\epsilon \frac{\partial}{\partial x_{t}}\left[\eta_{t}(x, t) P(x, t)\right] \\
& +\epsilon^{2} \frac{\partial^{2}}{\partial x_{t} \partial x_{t}}\left[P(x, t) \int_{0}^{t} \mathrm{~d} \tau K_{t}(x, t ; x, \tau)\right] \\
& -\epsilon^{2} \frac{\partial}{\partial x_{t}}\left[P(x, t) \int_{0}^{t} \mathrm{~d} \tau\left(\frac{\partial}{\partial u_{1}} K_{t}(u, t ; x, \tau)\right)_{u=x}\right]+C\left(\epsilon^{3}\right),
\end{aligned}
$$

where we have defined the averages as

$$
\eta_{t}(x, t) \equiv \overline{F_{1}(x, t)}
$$

and the covariances by

$$
K_{t /}(u, t ; x, \tau) \equiv \overline{F_{i}(u, t) F_{i}(x, \tau)}-\overline{F_{t}(u, t)} \overline{F_{i}(x, \tau)} .
$$

Multiplying eq. (A.20) by $x$, integrating over all possible values of the state variables. neglecting terms of order $\epsilon^{3}$ and higher, we obtain the following equation for the mean $\langle\boldsymbol{x}(t)\rangle$ :

$$
\frac{\mathrm{d}}{\mathrm{d} t}\left\langle x_{l}(t)\right\rangle=\epsilon\left\langle\eta_{l}(x(t), t)\right\rangle+\epsilon^{2} \int_{0}^{t} \mathrm{~d} \tau\left\langle\left(\frac{\partial}{\partial u} K_{l}(u, t ; x, \tau)\right)_{u=x}\right\rangle .
$$

where summation over repeated indices is understood.

\section{Appendix B}

In order to cast eqs. (20) and (24) in the format required in Stratonovich's theory, eq. (13), we shall notice that these equations can be written in the 
following vector form $[7,13]$ :

$$
\frac{\mathrm{d}}{\mathrm{d} t} \boldsymbol{Y}=\mathbf{A} \boldsymbol{Y}+\epsilon \mathbf{B}(t ; \varphi) \boldsymbol{Y}
$$

where $\mathbf{A}$ is a constant matrix; in the interaction representation

$$
\boldsymbol{x}=\mathrm{e}^{-\mathbf{A r}^{\prime} \boldsymbol{Y}}
$$

eq. (B.1) is written as

$$
\frac{\mathrm{d}}{\mathrm{d} t} \boldsymbol{x}=\epsilon \mathrm{e}^{-\mathrm{A} t} \mathrm{~B}(t ; \varphi) \mathrm{e}^{\mathrm{A} t} \boldsymbol{x}
$$

from where it follows that $F$ in eq. (13) is

$$
F[\boldsymbol{x}, t ; \varphi]=\mathbf{C}(t ; \varphi) \boldsymbol{x}
$$

where we have defined

$$
\mathbf{C}(t, \varphi) \equiv \mathrm{e}^{-\mathbf{A} t} \mathbf{B}(t ; \varphi) \mathrm{e}^{\mathbf{A} t}
$$

It can be shown that with $\boldsymbol{F}$ given by (B.4) the equations for the mean values in (A.23) can be written in vector form as

$$
\frac{\mathrm{d}}{\mathrm{d} t}\langle\boldsymbol{x}(t)\rangle=\epsilon \overline{\mathbf{C}(t)}\langle\boldsymbol{x}(t)\rangle+\epsilon^{2} \int_{0}^{t} \mathrm{~d} \tau[\overline{\mathbf{C}(t) \mathbf{C}(t-\tau)}]\langle x(t)\rangle:
$$

transforming back to the original variables, $Y$, we have that the equations for the mean values are given by

$$
\begin{aligned}
& \frac{\mathrm{d}}{\mathrm{d} t}\langle\boldsymbol{Y}(t)\rangle=\mathbf{A}\langle\boldsymbol{Y}(t)\rangle+\epsilon \overline{\mathbf{B}(t)}\langle\boldsymbol{Y}(t)\rangle \\
& +\epsilon^{2} \int_{0}^{t} \mathrm{~d} \tau \overline{\mathbf{B}(t) \mathrm{e}^{A_{\tau}} \mathbf{B}(t-\tau) \mathrm{e}^{-A_{\tau}}}\langle Y(t)\rangle .
\end{aligned}
$$

\section{Equation for the mean}

In order to obtain the equation for the mean, eq. (22), we define the state vector as

$$
\boldsymbol{Y} \equiv(Z, \dot{Z})^{+}
$$


such that eq. (20) can be written in the format of eq. (B.1), with

$$
A \equiv\left[\begin{array}{cc}
0 & 1 \\
-\omega_{0}^{2} & 0
\end{array}\right]
$$

and

$$
\mathbf{B}(t ; \varphi) \equiv\left[\begin{array}{cc}
0 & 0 \\
-\omega_{0}^{2} m(t ; \varphi) & 0
\end{array}\right],
$$

from where

$$
\exp \{\mathbf{A} \tau\}=\left[\begin{array}{cc}
\cos \left(\omega_{0} \tau\right) & \frac{1}{\omega_{0}} \sin \left(\omega_{01} \tau\right) \\
-\omega_{01} \sin \left(\omega_{0} \tau\right) & \cos \left(\omega_{11} \tau\right)
\end{array}\right] .
$$

Eq. (22) for the mean. $\langle Z(t)\rangle$, is obtained substituting the above expressions in eq. (B.7) and using the properties of the noise in eq. (21).

Equation for the mean square radius

In this case we define

$$
\boldsymbol{Y} \equiv\left(R^{2}, \frac{\mathrm{d}}{\mathrm{d} t} R^{2}, \frac{\mathrm{d}^{2}}{\mathrm{~d} t^{2}} R^{2}\right)^{+}
$$

such that eq. (24) can be cast in the form of eq. (B.1) defining

$$
\mathbf{A} \equiv\left[\begin{array}{ccc}
0 & 1 & 0 \\
0 & 0 & 1 \\
0 & -4 \omega_{0}^{2} & 0
\end{array}\right]
$$

and

$$
\mathbf{B}(t ; \varphi) \equiv\left[\begin{array}{ccc}
0 & 0 & 0 \\
0 & 0 & 0 \\
-2 \omega_{11}^{2} \dot{m}(t ; \varphi) & -4 \omega_{01}^{2} m(t ; \varphi) & 0
\end{array}\right]
$$

in this case

$$
\exp \{\mathbf{A} \tau\}=\left[\begin{array}{ccc}
1 & \frac{1}{2 \omega_{11}} \sin \left(2 \omega_{1,} \tau\right) & \frac{1}{4 \omega_{11}^{2}}\left[1-\cos \left(2 \omega_{11} \tau\right)\right] \\
0 & \cos \left(2 \omega_{1,} \tau\right) & \frac{1}{2 \omega_{10}} \sin \left(2 \omega_{1,} \tau\right) \\
0 & -2 \omega_{11} \sin \left(2 \omega_{1,} \tau\right) & \cos \left(2 \omega_{11} \tau\right)
\end{array}\right]
$$

Substituting the above equations in (B.7) and using eq. (21), we get after a little algebra the equation for the mean square radius, eq. (25). 


\section{References}

[1] R.B. Miller, An Introduction to the Physics of Intense Charged Particle Beams (Plenum, New York 1985).

[2] R.C. Davison. Relativistic Electron Beam-Plasma Interaction with Intense Self-Fields, Handbook of Plasma Physics, vol. 2, M.N. Rosenbluth and R.Z. Sagdeev, cds. (Elsevier, New York. 1984).

[3] N.G. van Kampen. Stochastic Processes in Physics and Chemistry (North-Holland, Amsterdam, 1981).

[4] H. Risken. Methods for Solving the Fokker-Planck Equation. Stochastic Processes Applied to Physics. L. Pesquera and M.A. Rodriguez, eds. (World Scientific, Singapore, 1985).

[5] N.G. van Kampen, J. Stat. Phys. 25 (1981) 431.

[6] A.Z. Akcasu, J. Stat. Phys. 16 (1977) 33.

(7) N.G. van Kampen, Phys. Rep. C 24 (1976) 171.

[s] K. Itô, Mem. Am. Math. Soc. 4 (1951) 51.

[9] R.L. Stratonovich. Topies in the Theory of Random No1se, vol. 1 (Gordon and Breach, New York. 1963).

[10] A.H. Gray Jr. and T.K. Caughey, J. Math. Phys. 44 (1965) 288.

[11] R.E. Mortensen. J. Stat. Phys. 1 (1969) 271.

[12] N.G. van Kampen. J. Stat. Phys. 24 (1981) 175.

[13] M.E. Fenstcrmacher. Ph.D. Thesis The University of Michigan (1983)

[14] C.T. Chen, Introduction to Linear System Theory (Holt, Rinehart and Winston. New York. 1970).

[15] A.Z. Akcasu and B. Hammouda. Physica A 131 (1985) 485.

[16] J. McKenna and J.A. Morrison, J. Math. Phys. 11 (1970) 2348. 